\title{
SASKATCHEWAN CHRISTMAS MAMMAL COUNT - 1992
}

Compiled by WAYNE C. HARRIS, Box 414, Raymore, Saskatchewan. SOA 3JO

As with Christmas bird counts the number of mammal counts dropped from last year's record high of 82 to 69 . The number of species recorded on these counts dropped from last year's high of 44 to a more normal 36. Species which have appeared on most of the counts in the past but which were absent from this year's count were Richardson's Ground Squirrel and Northern Pocket Gopher (undoubtedly due to the very cold weather). Woodland Caribou and American Marten were also absent.

A single new species was recorded on this year's count. An American Water Shrew was found on the snow covered ice adjacent to open water on the Waskesiu River in Prince Albert National Park. This is within the normal range of this species but it is rarely seen as it frequents the edges of fast flowing streams and is usually under the overhanging ice of the stream edge during the winter months.

Other rarely reported species include Lynx and Bobcat both from the Skull Creek count where tracks were seen; Eastern Cottontail on the Estevan count and Swift Fox from the Govenlock count.

With $97 \%$ of the counts having White-tailed Deer, they were the most common and the most frequently encountered species. Red Fox at $62 \%$ and Coyote at $58 \%$ were the next most frequent species. Hare populations appear to be remaining low with White-tailed Jack Rabbit with only $52 \%$ and Snowshoe Hare with $49 \%$ occurrences.

For weather, coverage and participants please refer to the Christmas Bird Count found elsewhere in this issue. Numbers appearing before the count location name in the tables refer to the location of the count on the map included with the bird count; some columns are left blank because no mammal counts were taken at those locations. In the tables a numeral alone indicates that the animals were actually seen, while a letter preceding the number indicates that the numbers were inferred by the means defined. A letter alone indicates that the species was present but it was either impossible to estimate numbers or no attempt was made to do so.

$\mathrm{T}=$ tracks

$L=$ active lodge or hut

$\mathrm{D}=$ dead animal found .

$\mathrm{d}=$ fresh diggings found

$\mathrm{S}=$ smell or odour

$\mathrm{H}=$ heard

$+=$ present during the count period (Dec. 17 to Jan. 3) but not found on count day. 


\begin{tabular}{|c|c|c|c|c|c|c|c|c|c|c|c|c|}
\hline & & & & & & $\angle O C A$ & TION AN & ID DATE & & & & \\
\hline SPECIES & 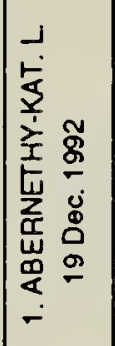 & 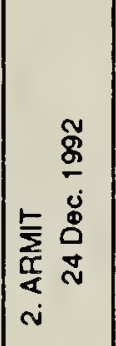 & 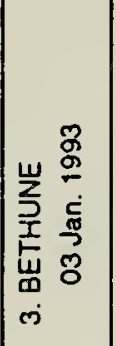 & 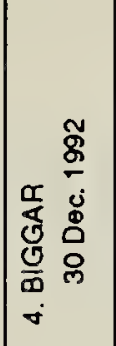 & 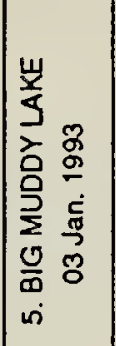 & 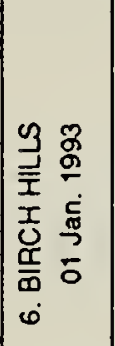 & 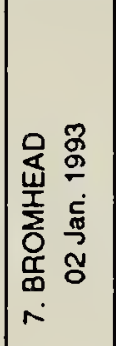 & 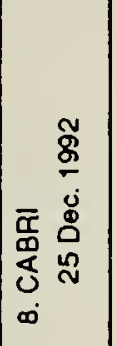 & 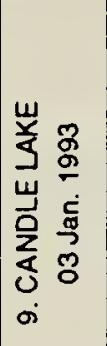 & 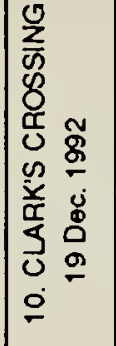 & 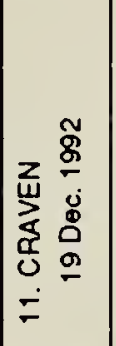 & 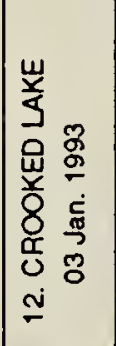 \\
\hline $\begin{array}{l}\text { MASKED SHREW } \\
\text { AMERICAN WATER SHREW } \\
\text { SHORT-TALLED SHAEW } \\
\text { SHREW SPECies } \\
\text { LITLE BROWN BAT }\end{array}$ & & & & & & & & & & & 1 & \\
\hline $\begin{array}{l}\text { EASTERN COTTONTALL } \\
\text { NUTTALL'S COTTONTALL } \\
\text { SNOWSHOEHARE } \\
\text { WHITE-TALED JACK RABBIT } \\
\text { RICHARDSON'S GROUND SOUIRREL }\end{array}$ & 1 & $\begin{array}{l}T \\
T\end{array}$ & & $\begin{array}{r}5 \\
T 15\end{array}$ & $\mathrm{~T}$ & $\begin{array}{l}T_{1} \\
T_{4}\end{array}$ & $T 14$ & $\begin{array}{l}1 \\
5\end{array}$ & & $\begin{array}{r}T 13 \\
3+T 14\end{array}$ & $\begin{array}{l}T_{16} \\
T_{12}\end{array}$ & $T$ \\
\hline $\begin{array}{l}\text { THIRTEEN-LINED GROUND SOUIRREL } \\
\text { BLACK.TALLED PRAIRIE DOG } \\
\text { GREY SOUIRREL } \\
\text { FOX SOUIRREL } \\
\text { AMERICAN RED SOUIRAEL }\end{array}$ & + & 7 & & & & $T_{1}$ & & & 3 & & & 1 \\
\hline $\begin{array}{l}\text { NORTHERN FLYING SOUIRRE } \\
\text { NORTHERN POCKET GOPHER } \\
\text { AMERICAN BEAVER } \\
\text { OEER MOUSE } \\
\text { GAPPER'S RED-BACKEOVOLE }\end{array}$ & & 12 & & & $T_{1}$ & & & & & & T9 & \\
\hline $\begin{array}{l}\text { MUSKRAT } \\
\text { MEADOWVOLE } \\
\text { VOLE Species } \\
\text { MOUSE Species } \\
\text { NORWAY RAT }\end{array}$ & & & & $\begin{array}{l}T 7 \\
T 5\end{array}$ & & T8 & & & & & & $T$ \\
\hline $\begin{array}{l}\text { HOUSE MOUSE } \\
\text { AMERICAN POACUPINE } \\
\text { COYOTE } \\
\text { WOLF } \\
\text { RED FOX }\end{array}$ & & $\begin{array}{r}1 \\
T 4 \\
T 3 \\
T 2\end{array}$ & & 10 & $\begin{array}{r}\mathrm{T} 2 \\
2 \\
1 \\
1\end{array}$ & $\begin{array}{r}1+T 5 \\
T 7\end{array}$ & 2 & & 1 & $T 1$ & $\begin{array}{c}1 \\
\mathrm{~T} 6 \\
\mathrm{~T} 8\end{array}$ & 2 \\
\hline $\begin{array}{l}\text { SMMFT FOX } \\
\text { RACCOON } \\
\text { AMERICAN MARTEN } \\
\text { FISHER } \\
\text { ERMINE } \\
\end{array}$ & & 12 & & & & 12 & & & & & & \\
\hline $\begin{array}{l}\text { LONG-TALLED WEASEL } \\
\text { LEAST WEASEL } \\
\text { WEASEL SPECCieS } \\
\text { AMERICAN MINK } \\
\text { AMERICAN BADGER }\end{array}$ & & T2 & & & $T_{1}$ & $\begin{array}{l}\mathrm{T} 2 \\
\mathrm{~T} 2\end{array}$ & & & & $T 3$ & T2 & $\begin{array}{l}T_{4} \\
T_{1}\end{array}$ \\
\hline $\begin{array}{l}\text { STAIPED SKUNK } \\
\text { RNER OTIEA } \\
\text { LYNX } \\
\text { BOBCAT } \\
\text { WOOOLAND CARIBOU }\end{array}$ & & & & & & & & & & & & \\
\hline $\begin{array}{l}\text { MULE DEER } \\
\text { WHITE-TALLED OEER } \\
\text { OEER SPECies } \\
\text { MOOSE } \\
\text { AMERICAN ELK }\end{array}$ & + & \begin{tabular}{r|}
4 \\
$\mathrm{~T} 2$ \\
$\mathrm{~T} 3$
\end{tabular} & $\begin{array}{l}+ \\
+\end{array}$ & $\begin{array}{l}1 \\
4\end{array}$ & $\begin{array}{l}22 \\
29\end{array}$ & $T 3$ & 23 & + & 3 & \begin{tabular}{|r}
6 \\
$3+\mathrm{T} 116$ \\
\end{tabular} & $\begin{array}{r}21 \\
T 26\end{array}$ & $T$ \\
\hline $\begin{array}{l}\text { PRONGHORN } \\
\text { TOTAL SPECIES }\end{array}$ & 4 & 13 & 2 & 8 & 92 & 11 & 31 & 3 & 3 & 7 & 10 & 7 \\
\hline
\end{tabular}




\begin{tabular}{|c|c|c|c|c|c|c|c|c|c|c|c|c|}
\hline & & & & & & LOCA & ATION AI & VD DATE & & & & \\
\hline SPECIES & 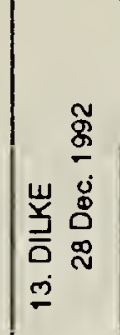 & 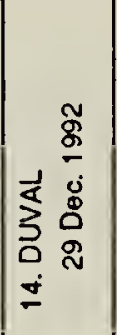 & 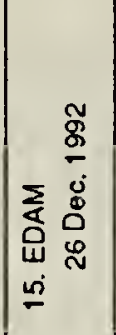 & 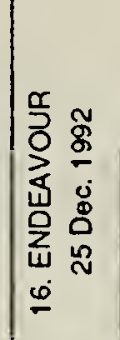 & 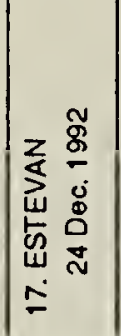 & 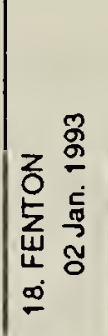 & 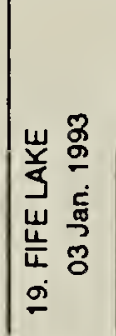 & 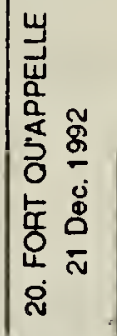 & 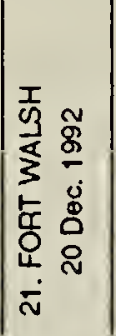 & 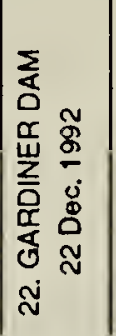 & 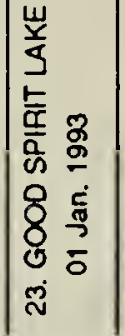 & 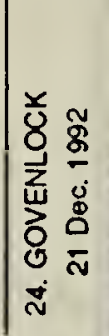 \\
\hline $\begin{array}{l}\text { MASKED SHREW } \\
\text { AMERICAN WATER SHREW } \\
\text { SHORT-TALLD SHREW } \\
\text { SHREW SPECies } \\
\text { LTTLE BROWN BAT }\end{array}$ & & & & & & & & & $\mathrm{T}_{1}$ & & & \\
\hline $\begin{array}{l}\text { EASTERN COTTONTAIL } \\
\text { NUTTALL'S COTTONTALL } \\
\text { SNOWSHOEHARE } \\
\text { WHITE-TALLED JACK RABBIT } \\
\text { RICHARDSON'S GROUND SOUIRRE }\end{array}$ & & & & & $\mathrm{T} 2$ & & & + & $\begin{array}{r}1 \\
T 1 \\
T 11\end{array}$ & $\begin{array}{l}\mathrm{T} 2 \\
\mathrm{~T} 2 \\
\mathrm{~T} 7\end{array}$ & $\begin{array}{l}\mathrm{T} 20 \\
\mathrm{~T} 22\end{array}$ & 2 \\
\hline $\begin{array}{l}\text { THIRTEEN-LINED GROUND SOUIRREL } \\
\text { BLACK-TALLD PRAIRIE DOG } \\
\text { GREY SOUIRRE. } \\
\text { FOX SOUIRREL } \\
\text { AMERICAN RED SOUIRRE }\end{array}$ & & & & 1 & $\mathrm{~T} 2$ & & & 3 & 41 & & T3 & \\
\hline $\begin{array}{l}\text { NORTHERN FLYING SOUIRRE } \\
\text { NORTHERN POCKET GOPHER } \\
\text { AMERICAN BEAVER } \\
\text { DEERMOUSE } \\
\text { GAPPER'S RED-BACKED VOLE }\end{array}$ & & 1 & & & & & & $\begin{array}{l}+ \\
1\end{array}$ & & & 1 & \\
\hline $\begin{array}{l}\text { MUSKRAT } \\
\text { MEADOWVOLE } \\
\text { VOLE Species } \\
\text { MOUSE Species } \\
\text { NORWAY RAT }\end{array}$ & & $\mathrm{T} 2$ & & & & & & + & $\mathrm{T} 8$ & $T 11$ & $L 1$ & $\begin{array}{r}T 10 \\
T\end{array}$ \\
\hline $\begin{array}{l}\text { HOUSE MOUSE } \\
\text { AMERICAN PORCUPINE } \\
\text { COYOTE } \\
\text { WOLF } \\
\text { RED FOX }\end{array}$ & & $\begin{array}{l}\mathrm{T} 1 \\
\mathrm{~T} 5\end{array}$ & & $T 1$ & $\mathrm{~T} 1$ & & & + & $\begin{array}{r}1 \\
16\end{array}$ & $\mathrm{~T} 2$ & $\begin{array}{r}\mathrm{T} 3 \\
3 \\
\mathrm{~T} 2\end{array}$ & $\begin{array}{l}\mathrm{T}_{1} \\
12\end{array}$ \\
\hline $\begin{array}{l}\text { SWMFT FOX } \\
\text { RACCOON } \\
\text { AMERICAN MARTEN } \\
\text { FISHER } \\
\text { ERMINE }\end{array}$ & & & & & & & & & & & T5 & + \\
\hline $\begin{array}{l}\text { LONG TAILED WEASEL } \\
\text { LEAST WEASEL } \\
\text { WEASEL SPECies } \\
\text { AMERICAN MINK } \\
\text { AMERICAN BADGER }\end{array}$ & & & & & & & & + & $\begin{array}{l}T_{1} \\
T_{1}\end{array} \mid$ & $\mathrm{T} 2$ & $T_{1}$ & de \\
\hline $\begin{array}{l}\text { STRIPED SKUNK } \\
\text { RNER OTTER } \\
\text { LYNX } \\
\text { BOBCAT } \\
\text { WOODLAND CARIBOU }\end{array}$ & & & & & & & & & & & & \\
\hline $\begin{array}{l}\text { MULE DEER } \\
\text { WHITE.TALED DEER } \\
\text { DEER Species } \\
\text { MOOSE } \\
\text { AMERICAN ELK }\end{array}$ & & T3 & & $\pi_{1}$ & 2 & & & + & $\begin{array}{r}107 \\
80 \\
\\
\mathrm{~T} 1 \\
\mathrm{~T} 15 \\
\end{array}$ & $\begin{array}{l}14 \\
47\end{array}$ & $\begin{array}{c}\mathrm{T} 37 \\
\mathrm{~T} 1\end{array}$ & $\begin{array}{r}121 \\
35\end{array}$ \\
\hline $\begin{array}{l}\text { PRONGHORN } \\
\text { TOTAL SPECIES }\end{array}$ & 이 & 5 & 0 & 4 & 5 & 1 & 0 & 11 & 16 & 9 & 13 & $\begin{array}{r}116 \\
13 \\
\end{array}$ \\
\hline
\end{tabular}




\begin{tabular}{|c|c|c|c|c|c|c|c|c|c|c|c|c|}
\hline & & & & & & LOCA & TION AN & ID DATE & & & & \\
\hline SPECIES & 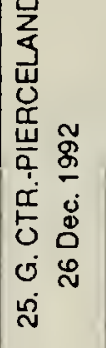 & 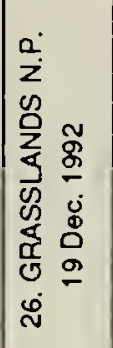 & 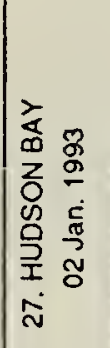 & 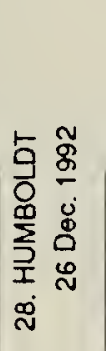 & 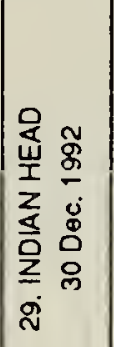 & 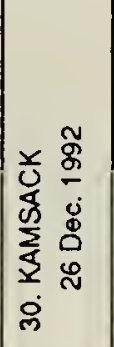 & 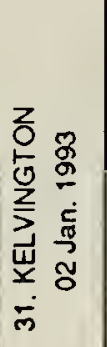 & 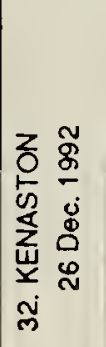 & 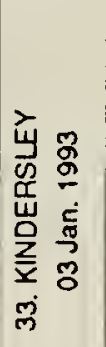 & 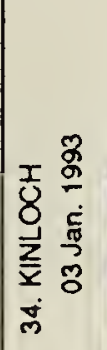 & 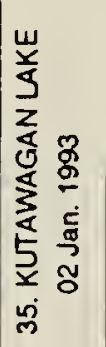 & 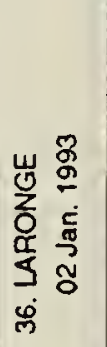 \\
\hline $\begin{array}{l}\text { MASKED SHREW } \\
\text { AMERICAN WATER SHREW } \\
\text { SHORT-TAILED SHREW } \\
\text { SHREW SPECIES } \\
\text { LTTLE BROWN BAT }\end{array}$ & & & & & & & & & & & & \\
\hline $\begin{array}{l}\text { EASTERN COTTONTAIL } \\
\text { NUTTALL'S COTTONTAIL } \\
\text { SNOWSHOE HARE } \\
\text { WHITE-TAILED JACK RABBIT } \\
\text { RICHARDSON'S GROUND SOUIRREL }\end{array}$ & $T 13$ & 1 & & $\begin{array}{r}1 \\
T_{1}\end{array}$ & $5+T_{12}$ & 26 & $\begin{array}{l}T_{4} \\
T_{2}\end{array}$ & & & & $T$ & $\mathrm{~T} 2$ \\
\hline $\begin{array}{l}\text { THIRTEEN-LINED GROUND SOUIRAEL } \\
\text { BLACK-TAILED PRAIRIE DOG } \\
\text { GREY SOUIRREL } \\
\text { FOX SOUIRREL } \\
\text { AMERICAN RED SOUIRREL }\end{array}$ & 1 & & & & 1 & & & & & & & 3 \\
\hline $\begin{array}{l}\text { NORTHEAN FLYING SOUIRREL } \\
\text { NORTHERN POCKET GOPHER } \\
\text { AMERICAN BEAVER } \\
\text { DEEAMOUSE } \\
\text { GAPPER'S RED-BACKED VOLE }\end{array}$ & & $T$ & & & & & & & & & & \\
\hline $\begin{array}{l}\text { MUSKRAT } \\
\text { MEEADOW VOLE } \\
\text { VOLE species } \\
\text { MOUSE species } \\
\text { NORWAY RAT }\end{array}$ & & 10 & & & & & & $T$ & & & & $T_{1}$ \\
\hline $\begin{array}{l}\text { HOUSE MOUSE } \\
\text { AMERICAN PORCUPINE } \\
\text { COYOTE } \\
\text { WOLF } \\
\text { RED FOX } \\
\end{array}$ & + & $\begin{array}{l}6 \\
4\end{array}$ & & & + & 7 & $T_{1}$ & $T$ & & & 1 & $\begin{array}{l}\mathrm{T} 5 \\
\mathrm{~T} 3 \\
\end{array}$ \\
\hline $\begin{array}{l}\text { SWIFT FOX } \\
\text { RACCOON } \\
\text { AMERICAN MARTEN } \\
\text { FISHER } \\
\text { ERMINE }\end{array}$ & & & & & & & & & & & & $\mathrm{T}_{3}$ \\
\hline $\begin{array}{l}\text { LONG-TALED WEASEL } \\
\text { LEAST WEASEL } \\
\text { WEASEL SPECies } \\
\text { AMERICAN MINK } \\
\text { AMERICAN BADGER }\end{array}$ & & & & & $\mathrm{T} 2$ & & $\begin{array}{l}T_{1} \\
T_{1}\end{array}$ & & & & $T$ & \\
\hline $\begin{array}{l}\text { STRIPED SKUNK } \\
\text { RIVER OTTER } \\
\text { LYNX } \\
\text { BOBCAT } \\
\text { WOODLAND CARIBOU }\end{array}$ & & & & & + & & & & & & & \\
\hline $\begin{array}{l}\text { MULE DEEA } \\
\text { WHITE-TAILED DEER } \\
\text { DEER species } \\
\text { MOOSE } \\
\text { AMERICAN ELK }\end{array}$ & $T_{15}$ & $\begin{array}{r}111 \\
67\end{array}$ & & T3 & + & $\begin{array}{r}2 \\
50\end{array}$ & 6 & $T$ & 9 & & $T$ & \\
\hline $\begin{array}{l}\text { PRONGHORN } \\
\text { TOTAL SPECIES }\end{array}$ & 4 & 444 & 0 & 3 & 7 & 5 & 7 & 3 & 1 & 0 & 5 & 6 \\
\hline
\end{tabular}




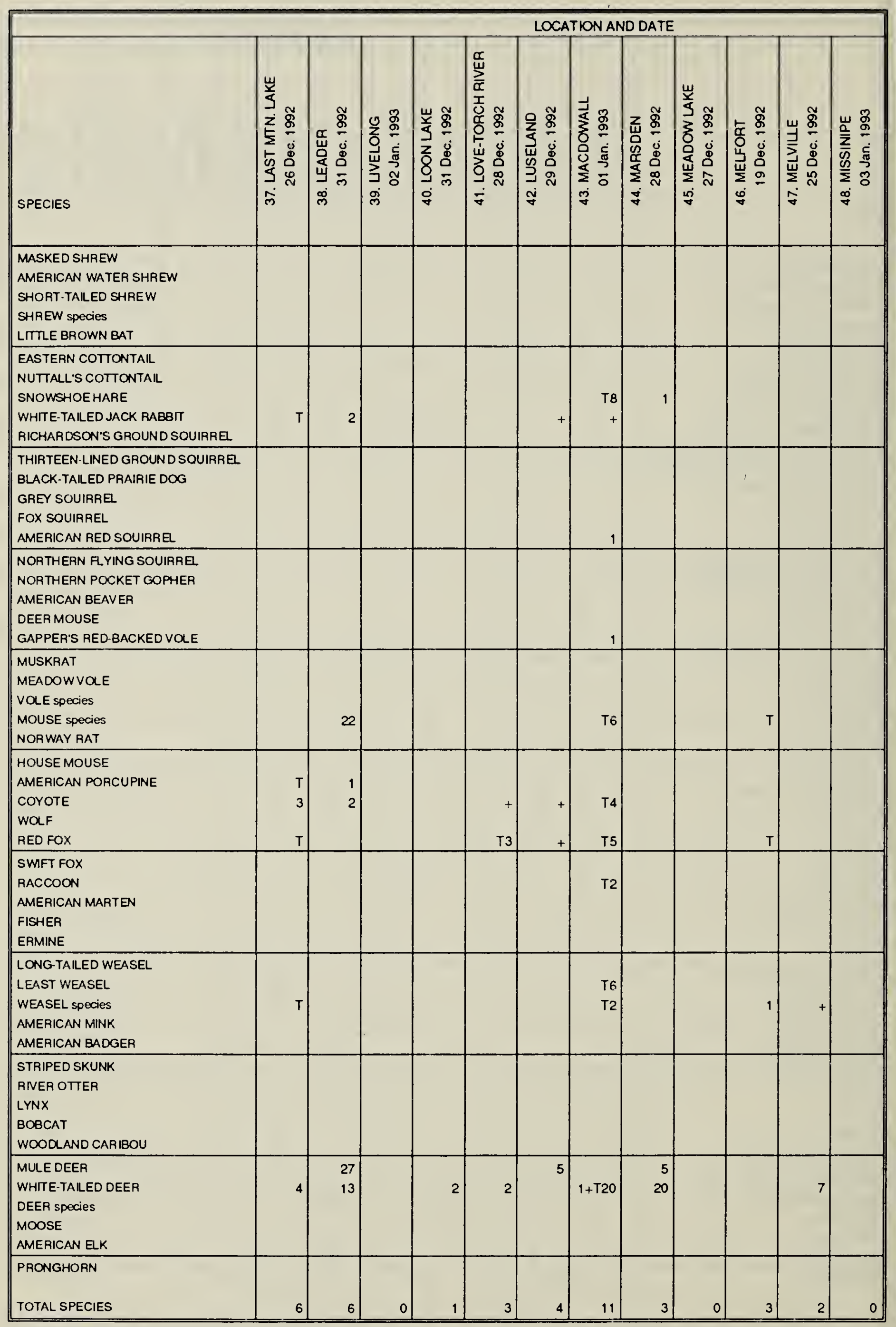




\begin{tabular}{|c|c|c|c|c|c|c|c|c|c|c|c|c|}
\hline & & & & & & $\angle O C A$ & TION AI & ID DATE & & & & \\
\hline SPECIES & 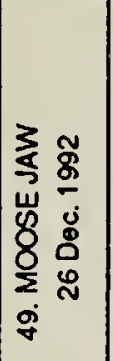 & 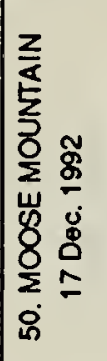 & 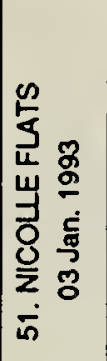 & 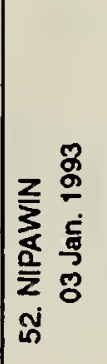 & 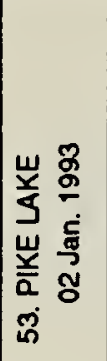 & 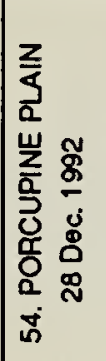 & 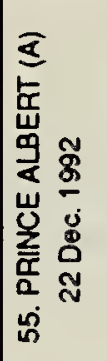 & 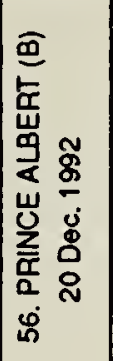 & 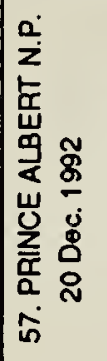 & 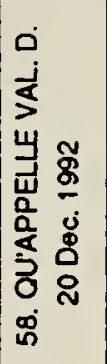 & 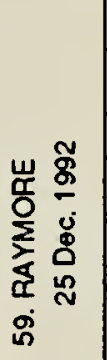 & 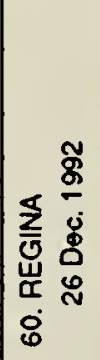 \\
\hline $\begin{array}{l}\text { MASKED SHREW } \\
\text { AMERICAN WATER SHREW } \\
\text { SHORT-TAILED SHREW } \\
\text { SHREW SPECies } \\
\text { LTME BROWN BAT }\end{array}$ & & & & & & & & & + & & 1 & . \\
\hline $\begin{array}{l}\text { EASTERN COTTONTAIL } \\
\text { NUTTALL'S COTTONTAIL } \\
\text { SNOWSHOE HARE } \\
\text { WHITE-TAILED JACK RABBIT } \\
\text { RICHARDSON'S GROUND SOUIRREL }\end{array}$ & & & D1 & & $\begin{array}{l}\text { T5 } \\
\text { T5 }\end{array}$ & & & & T8 & $1+T$ & $\begin{array}{l}\text { T5 } \\
\text { T3 }\end{array}$ & $\begin{array}{l}2 \\
3\end{array}$ \\
\hline $\begin{array}{l}\text { THIRTEEN-LINED GROUND SOUIRRE } \\
\text { BLACK-TALED PRAIRIE DOG } \\
\text { GREY SOUIRRE } \\
\text { FOX SOUIRREL } \\
\text { AMERICAN RED SOUIRREL }\end{array}$ & 2 & 3 & & & & & & 1 & $1+T 5$ & & & 1 \\
\hline $\begin{array}{l}\text { NORTHERN FYYING SOUIRRE } \\
\text { NORTHERN POCKET GOPHER } \\
\text { AMERICAN BEAVER } \\
\text { DEERMOUSE } \\
\text { GAPPER'S REDBACKED VOLE }\end{array}$ & & & & & 1 & & & & T1 & & $\begin{array}{r}\mathrm{L} 1 \\
2 \\
1\end{array}$ & \\
\hline $\begin{array}{l}\text { MUSKRAT } \\
\text { MEADOW VOLE } \\
\text { VOLE SPECies } \\
\text { MOUSE SPECIeS } \\
\text { NORWAY RAT }\end{array}$ & & & & & & & & & & & \begin{tabular}{r|} 
L1 \\
2 \\
T2 \\
T7
\end{tabular} & $\mathrm{L} 2$ \\
\hline $\begin{array}{l}\text { HOUSE MOUSE } \\
\text { AMERICAN PORCUPINE } \\
\text { COYOTE } \\
\text { WO.F } \\
\text { RED FOX }\end{array}$ & & 1 & 1 & & & & & & $\begin{array}{r}\mathrm{T} 1 \\
\mathrm{~T} 2 \\
3+6 \mathrm{~T}\end{array}$ & $\begin{array}{r}3+2 T \\
1\end{array}$ & $\begin{array}{l}2 \\
2 \\
4\end{array}$ & 8 \\
\hline $\begin{array}{l}\text { SWFT FOX } \\
\text { RACCOON } \\
\text { AMERICAN MARTEN } \\
\text { FISHER } \\
\text { ERMINE }\end{array}$ & & & & & & & & & 1 & & & \\
\hline $\begin{array}{l}\text { LONG-TAILED WEASEL } \\
\text { LEAST WEASEL } \\
\text { WEASEL SPECieS } \\
\text { AMERICAN MINK } \\
\text { AMERICAN BADGER }\end{array}$ & & & & & & & & & 1 & & $\begin{array}{r}+ \\
\text { T5 }\end{array}$ & \\
\hline $\begin{array}{l}\text { STRIPED SKUNK } \\
\text { RNER OTTER } \\
\text { LYNX } \\
\text { BOBCAT } \\
\text { WOODLAND CARIBOU } \\
\end{array}$ & & & & & & & & & T4 & & & \\
\hline $\begin{array}{l}\text { MULE DEER } \\
\text { WHITE-TAILED DEER } \\
\text { DEER species } \\
\text { MOOSE } \\
\text { AMERICAN EKK }\end{array}$ & 3 & 8 & $\begin{array}{r}6 \\
39\end{array}$ & & 2 & & & 9 & $\begin{array}{r}\mathrm{T} 8 \\
\mathrm{~T} 1 \\
\mathrm{~T} 2 \\
3+45 \mathrm{~T}\end{array}$ & $\begin{array}{r}5 \\
15\end{array}$ & 7 & $\begin{array}{r}13 \\
T\end{array}$ \\
\hline $\begin{array}{l}\text { PRONGHORN } \\
\text { TOTAL SPECIES }\end{array}$ & 2 & 3 & 5 & 0 & 4 & 0 & 0 & 2 & 14 & 5 & 17 & 7 \\
\hline
\end{tabular}




\begin{tabular}{|c|c|c|c|c|c|c|c|c|c|c|c|c|}
\hline & & & & & & LOCA & TION AI & ID DATE & & & & \\
\hline SPECIES & 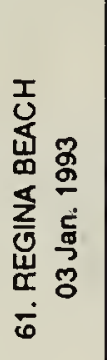 & 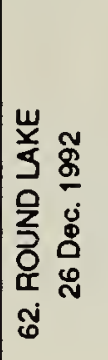 & 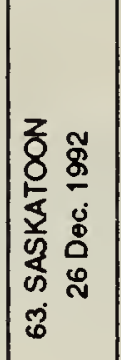 & 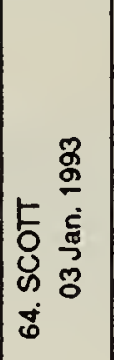 & 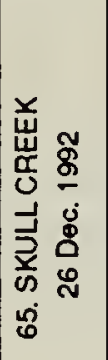 & 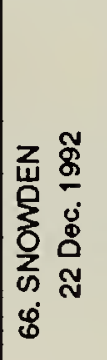 & 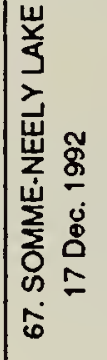 & 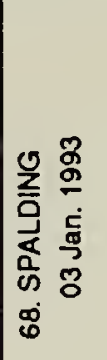 & 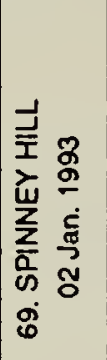 & 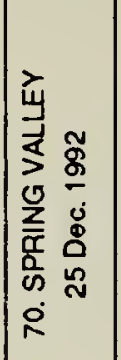 & 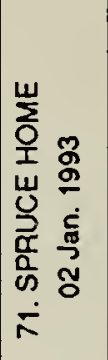 & 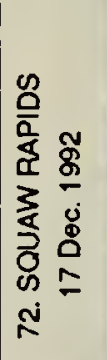 \\
\hline $\begin{array}{l}\text { MASKED SHREW } \\
\text { AMERICAN WATER SHREW } \\
\text { SHORT-TAILED SHREW } \\
\text { SHREW SPECIES } \\
\text { LTTLE BROWN BAT }\end{array}$ & & & & & $T$ & & & & $T$ & & & \\
\hline $\begin{array}{l}\text { EASTERN COTTONTAIL } \\
\text { NUTTALL'S COTTONTAIL } \\
\text { SNOWSHOE HARE } \\
\text { WHITE-TAILED JACK RABBIT } \\
\text { RICHARDSON'S GROUND SOUIRRE } \\
\end{array}$ & $\begin{array}{l}1 \\
1\end{array}$ & $\begin{array}{l}\mathrm{T} 1 \\
\mathrm{~T} 1\end{array}$ & $2+21 T$ & 7 & 11 & T9 & & $\mathrm{T}$ & $T$ & $2+T$ & & $\begin{array}{r}T 19 \\
T\end{array}$ \\
\hline $\begin{array}{l}\text { THIRTEEN-LINED GROUND SOUIRRE } \\
\text { BLACK-TAILED PRAIRIE DOG } \\
\text { GREY SOUIRREL } \\
\text { FOX SOUIRREL } \\
\text { AMERICAN RED SOUIRREL }\end{array}$ & & & & & 2 & 2 & & & & & & 10 \\
\hline $\begin{array}{l}\text { NORTHERN RLYING SOUIRRE } \\
\text { NORTHERN POCKET GOPHER } \\
\text { AMERICAN BEAVER } \\
\text { DEERMOUSE } \\
\text { GAPPER'S RED-BACKED VOE }\end{array}$ & & T1 & & & & & & & & & & L2 \\
\hline $\begin{array}{l}\text { MUSKRAT } \\
\text { MEADOWVOLE } \\
\text { VOLE Species } \\
\text { MOUSE Species } \\
\text { NORWAY RAT } \\
\end{array}$ & & T1 & & $T 7$ & $T$ & T6 & & $\begin{array}{l}T \\
T\end{array}$ & $T$ & $T$ & & $\begin{array}{l}1 \\
T\end{array}$ \\
\hline $\begin{array}{l}\text { HOUSE MOUSE } \\
\text { AMERICAN PORCUPINE } \\
\text { COYOTE } \\
\text { WOF } \\
\text { RED FOX } \\
\end{array}$ & $\begin{array}{l}\mathrm{T} 1 \\
\mathrm{~T} 2 \\
\mathrm{~T} 3 \\
\end{array}$ & & $\begin{array}{l}1 \\
2 \\
\end{array}$ & $\begin{array}{l}1 \\
2\end{array}$ & $\begin{array}{l}6 \\
7\end{array}$ & $\begin{array}{l}17 \\
\mathrm{~T} 2 \\
\end{array}$ & & & $\begin{array}{r}T \\
2+T \\
T \\
\end{array}$ & $\begin{array}{l}T \\
3 \\
1 \\
\end{array}$ & & $\begin{array}{r}T 1 \\
2 \\
T 1 \\
1 \\
\end{array}$ \\
\hline $\begin{array}{l}\text { SWFT FOX } \\
\text { RACCOON } \\
\text { AMERICAN MARTEN } \\
\text { FISHER } \\
\text { ERMINE }\end{array}$ & & & $T$ & & & T1 & & & & & & T \\
\hline $\begin{array}{l}\text { LONG-TAILED WEASEL } \\
\text { LEAST WEASEL } \\
\text { WEASEL SPECies } \\
\text { AMERICAN MINK } \\
\text { AMERICAN BADGER }\end{array}$ & & $\mathrm{T}_{1}$ & $T$ & & $\begin{array}{l}T \\
1\end{array}$ & $\begin{array}{l}\mathrm{T} 2 \\
\mathrm{~T} 2\end{array}$ & & $T$ & & & & $T$ \\
\hline $\begin{array}{l}\text { STRIPED SKUNK } \\
\text { RNER OTTER } \\
\text { LYNX } \\
\text { BOBCAT } \\
\text { WOODLAND CARIBOU }\end{array}$ & & & & & $\begin{array}{l}T \\
T\end{array}$ & & & & & & & \\
\hline $\begin{array}{l}\text { MULE DEER } \\
\text { WHITE-TAILED DEER } \\
\text { DEER Species } \\
\text { MOOSE } \\
\text { AMERICAN ELK } \\
\end{array}$ & $4+3 T$ & & $11+15 T$ & T5 & $\begin{array}{l}23 \\
43\end{array}$ & T3 & & $T$ & $\begin{array}{r}1+T \\
T \\
T\end{array}$ & 8 & + & $\begin{array}{r}4 \\
T 3 \\
T 10 \\
\end{array}$ \\
\hline $\begin{array}{l}\text { PRONGHORN } \\
\text { TOTAL SPECIES }\end{array}$ & 6 & 6 & 6 & 5 & 17 & 10 & 0 & 5 & 9 & 6 & 1 & 15 \\
\hline
\end{tabular}


